\title{
Exothermic Self-sustained Waves with Amorphous Nickel
}

Khachatur V. Manukyan ${ }^{1}$, Christopher E. Shuck ${ }^{2}$, Mathew J. Cherukara ${ }^{3}$, Sergei Rouvimov ${ }^{4}$,

Dmitry Y. Kovalev ${ }^{5}$, Alejandro Strachan ${ }^{3}$, and Alexander S. Mukasyan ${ }^{2.6}$

Departments of Physics ${ }^{1}$, Chemical \& Biomolecular Engineering ${ }^{2}$ and Electrical Engineering ${ }^{4}$ University of Notre Dame, Notre Dame, Indiana 46556, United States

${ }^{3}$ School of Mechanical Engineering and School of Materials Engineering,

Purdue University, West Lafayette, Indiana 47907, United States

${ }^{5}$ Institute of Structural Macrokinetics and Materials Science, Russian Academy of Sciences,

Chernogolovka, Moscow Region, 142432, Russia

${ }^{6}$ National University of Science and Technology, "MISIS", Moscow 119049, Russia

\section{Supporting information}



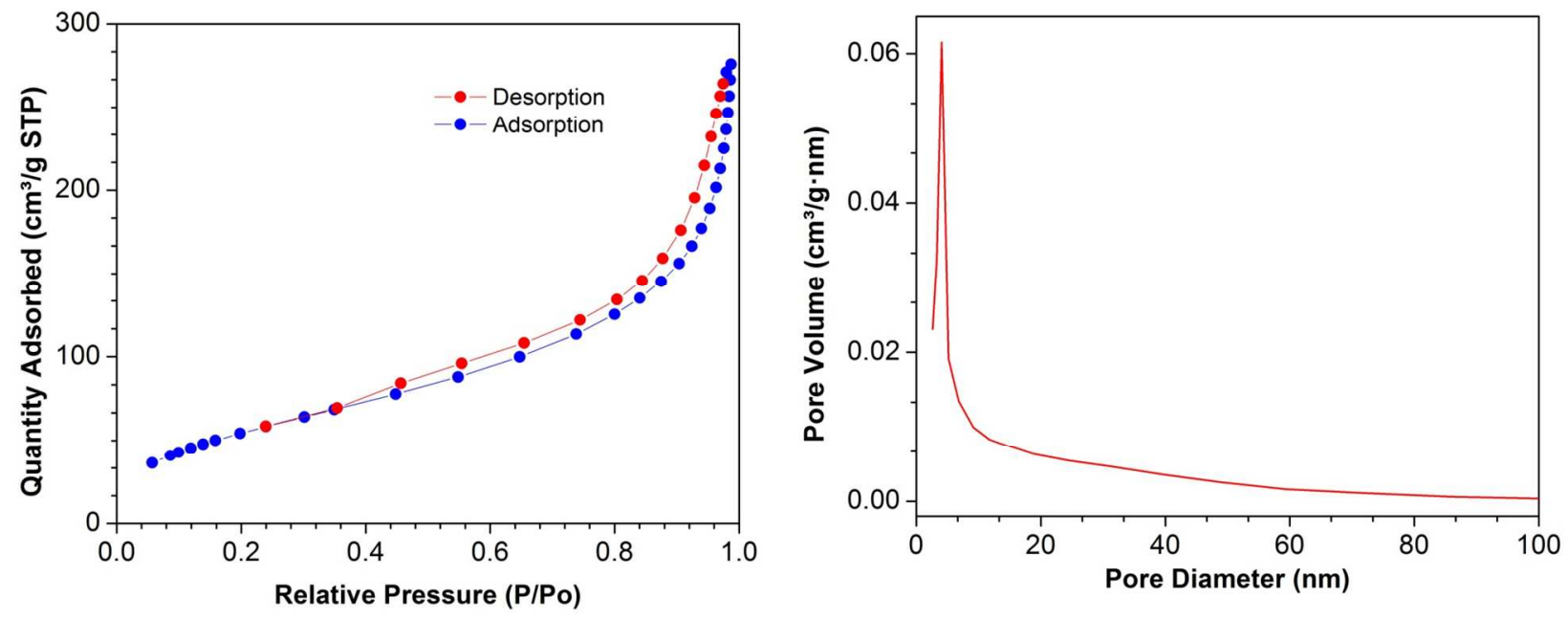

Figure S1 The results of nitrogen adsorption-desorption analysis of and calculated pore size distribution of a-Ni 

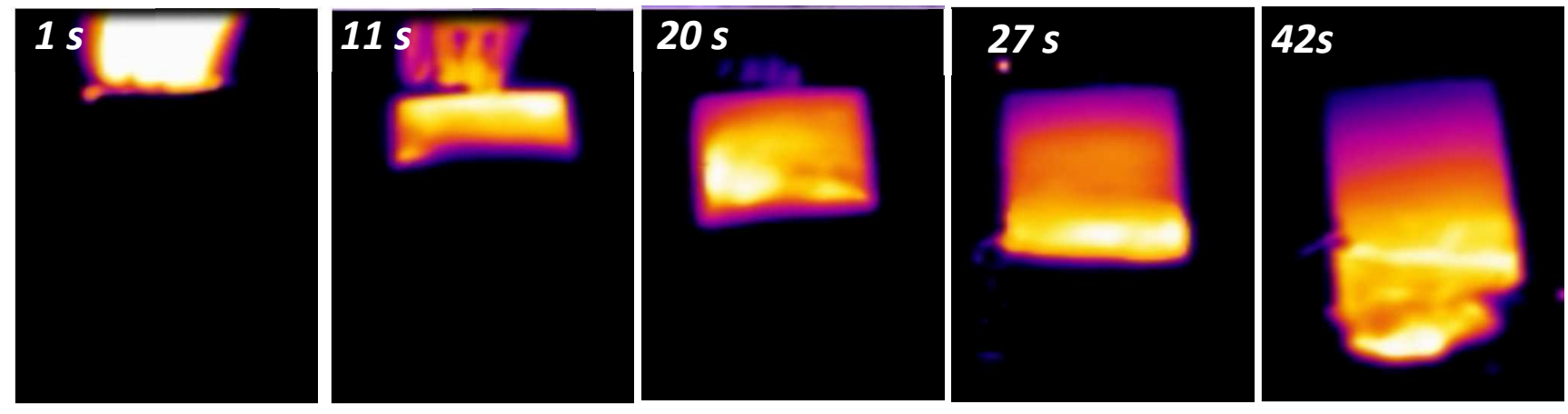

Figure S2: Snapshots of infrared imaging self-propagating crystallization. 

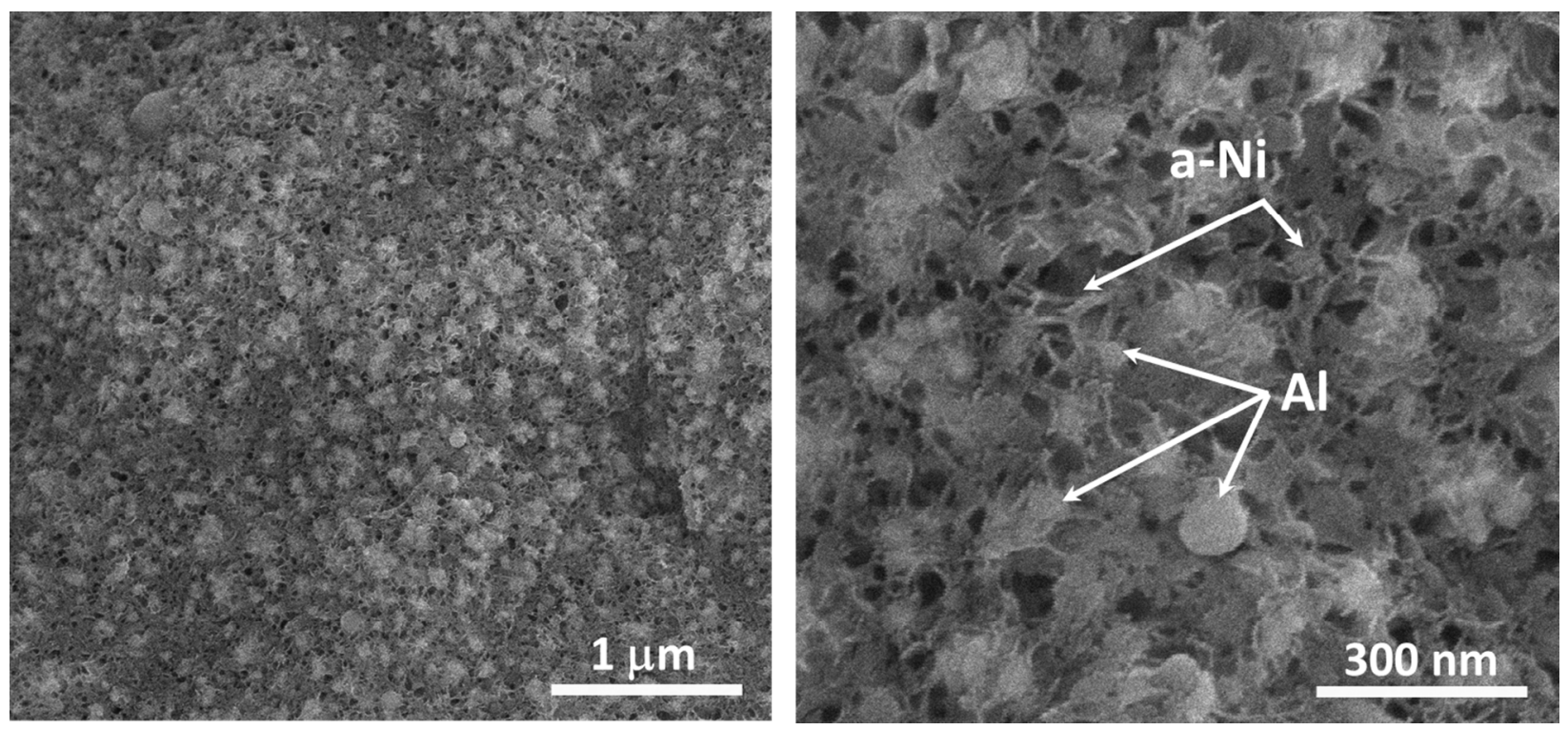

Figure S3: SEM images of a-Ni mixture with Al nanopowder 

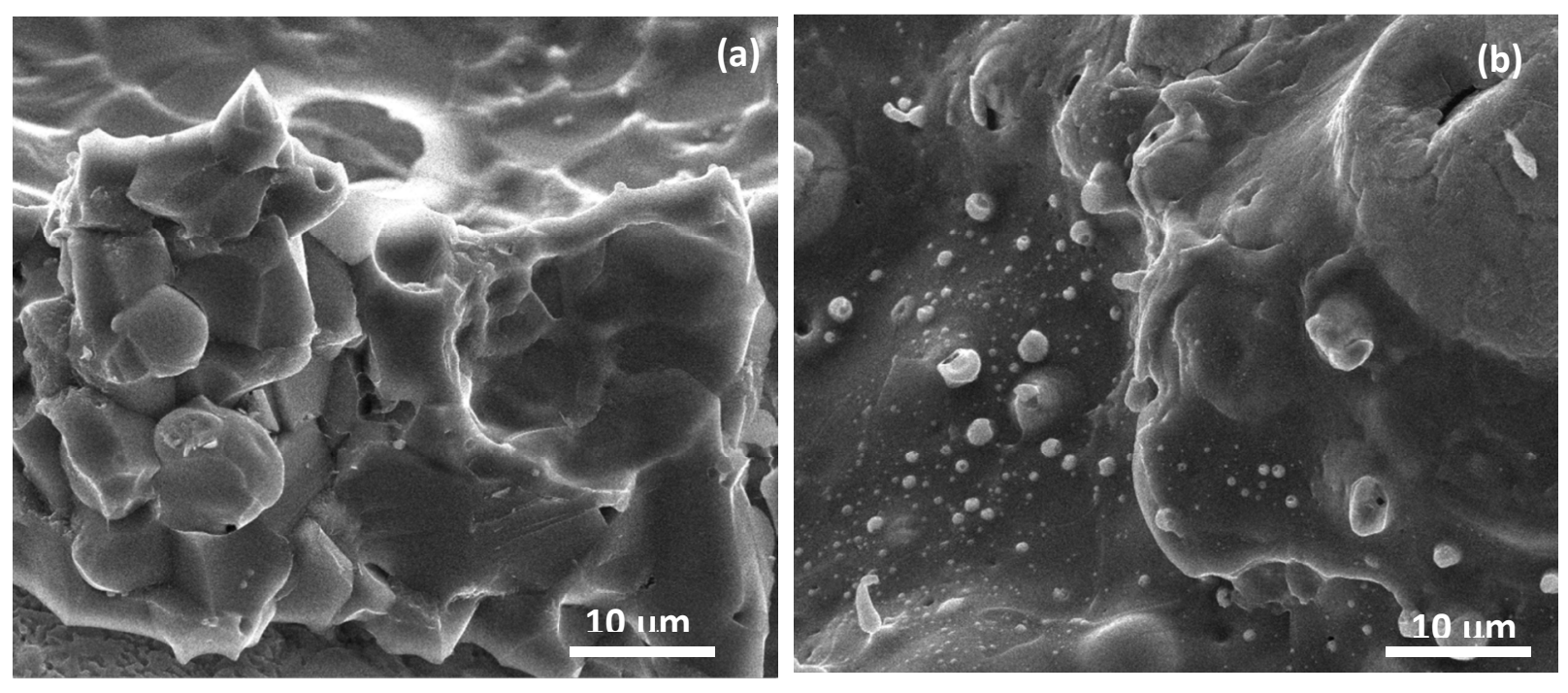

Figure S4: SEM images of NiAl products obtained by combustion of $\mathrm{a}-\mathrm{Ni}+\mathrm{Al}(\mathrm{a})$ and c-Ni+Al (b) samples 